International Journal of Engineering \& Technology, $7(4.38)(2018) 988-991$
International Journal of Engineering \& Technology
Website: $w w w . s c i e n c e p u b c o . c o m / i n d e x . p h p / I J E T$
Research paper

\title{
Comprehending Crisis Intervention among Helpers
}

\author{
Nor Shafrin Ahmad1, Siti Nur Nadhirah Mohd Ashri \\ School of Educational Studies \\ 11800, Universiti Sains Malaysia, \\ Pulau Pinang
}

\begin{abstract}
Crisis applies when an individual experiences a trauma or tense situation that causes imbalance, disorganization and disorientation. Failure to oversee the crisis brings a negative impression to the whole self. This study involved 25 crisis helpers as respondents and all of them are registered counselors in Malaysia. Data are collected through semi structured interviews. The open questions in semi structured interview were used to identify information on 1 . the meaning of crisis, 2. the process used to handle crisis intervention; 3 . the model of crisis intervention used among helpers. Results showed that respondents' comprehending of crisis were quite accurate and varied depending on situations experienced. They also had some brief ideas on how the process should be implemented to handle crisis cases. However, only nine out of 25 helpers specifically mentioned that they used models such as Roberts's seven stages, Gilliland six steps, Kubler Ross, Critical Incident Stress Debriefing to handle crisis intervention cases. Meanwhile, 19 helpers used counseling theories such as Cognitive Behavioral Therapy (CBT), Reality Therapy (RT), Psychological First Aid (PFA) and basic attending counseling skills. This study indicates that helpers need more knowledge and training on crisis intervention. They also need to comprehend process to handle crisis intervention cases in appropriate and structured ways. Development of a crisis intervention model that suits Malaysia's culture would be most preferable and later utilized as a guide for helpers and counselors in dealing with event of crisis.
\end{abstract}

Keywords: Crisis intervention, Robert's seven stage crisis intervention model, ABC crisis intervention model

\section{Introduction}

Crisis could happen to anyone throughout their daily life as an unexpected traumatic event. Samsiah Mohd Jais (2016), stated that crisis occurs when the traumatic event is perceived as overwhelming by the individual. Therefore, the resilience and coping mechanisms taken could differ from one individual to another; it is based on one's perceptions and acceptance.

There are several types of crisis that might occur. According to James and Gililland (2005), the four categories of crisis experienced by humans are 1. developmental (for example pension), 2. traumatic events (for example abuse), 3. existence (for example intrapersonal conflict) and 4. systemic (for example natural disasters). In Malaysia a number of crisis had taken place and these immense crisis started with the ruins of highland towers, tsunami in Penang, lost of MH370, the death of MH17 passengers, a major flood disaster in Kelantan at the end of 2014, and a flood disaster in Penang on December 2017. Crisis sometimes also happened to us and family such as involved in an accident, death of family members, divorce, being the victims of robbery and snatch theft, being shot, being rape, victim of domestic violence, attempted murder and various other distressing circumstances. All these conditions are the source of crisis of self either involving individuals as victims of crisis or as observers or witnesses involved in the incident.

According to Roberts and Yeager (2009), crisis is a subjective reaction to a stressful or traumatic life event or a series of events that are perceived by the person as hazardous, threatening or extremely upsetting, which cannot be resolved using traditional coping methods. It is important for helpers especially counselors to be prepared with fitting models as a guide for them to help individuals in crisis. The models need to be adapted to various cultures and approved by the society.

While crisis happened, crisis intervention used to handle the situation. Crisis intervention is one of the effort to help victims that having psychology difficulties to prevent traumatic negative effect on crisis (Everly \& Mitchell, 1999; Nor Shafrin Ahmad, 2009). According to Greenstone and Leviton, (2002), the act of interrupting the downward spiral as skillfully and as quickly as possible, to return the victim to a pre-crisis level of coping is called crisis intervention.

There are six major components in crisis intervention highlighted by Greenstone and Leviton (2011). Firstly is immediacy which means for the helpers to act immediately to stop the emotional bleeding. The helpers must respond immediately to relieve anxiety, prevent further disorientation and to ensure that there are no further harm could occur. The second components are the helpers have to take control and be clear about the situation and persons involved in the crisis by approaching the crisis event cautiously.

The appearance of helpers as stable, supportive and calm in the crisis may exert the control that the victim needs. The third component stated in crisis intervention is to assess the situation by doing some evaluations of what exactly happened and to comprehend the victim's perceptions towards the event.

Deciding how to handle the situation after the assessment is the next component that needs to be established. It is important for the helpers to help the victim identify and mobilize personal resources and develop options by thinking creatively. The next component would be to refer the victims as needed. The helpers have to check with professionals such as doctors, lawyers and counselors in the community. By making a list of acceptable referral resources, the helpers can refer the victim in the right place. The last component 
in crisis intervention as suggested by Greenstone and Leviton (2011) is to follow-up with the victims to ensure that they have made contact with the referral agency. All of the six components of a crisis intervention have to be employed to ensure the victims are not neglected.

Four attributes as components needed in helpers and other crisis interveners who intervene victims in the multicultural background. They need to have self-knowledge, knowledge about the statues and cultures of different groups, skills to effect culturally appropriates and actual experience in crisis intervention with multicultural victims (Kiselica, 1998 ; James 2008).

According to a research by Myer and Conte (2006), the triage assessment system (TAS) for crisis intervention was a model specifically designed for crisis is needed to guide the assessment process. TAS presumes that reaction to crisis events in three domains: 1. affective, 2. behavioral, and 3. cognitive. The results shows that TAS provides a quick and accurate that is directly usable in the intervention process.

Crisis management may differ from country based on different situation and variation of culture. Each of country needs to be analyzed from cultural dimensions and legal aspects for appropriate action plan (Ehsan Khidarahmi, 2009). In order to construct a model for crisis intervention the researcher need to determine the level of comprehending and knowledge from the potential helpers to make sure the crisis intervention model would be suitable and applicable to be implement. Therefore, by exploring and using all the elements that been needed for the helpers to intervene in crisis intervention, a suitable model that suits with multicultural varies might potentially help the victims immediately.

\section{Models in crisis intervention}

\subsection{Roberts' Seven Stage Crisis Intervention Model}

It is important for the helpers to have a stable and efficient model for crisis intervention. One of the models that has been used is the seven-stage crisis intervention model. Roberts and Yeager (2009), has identified seven critical stages typically used by the helpers to ensure the victims are stabilized when faced with a crisis. The seven stages are:

Stage 1: Psychosocial and lethality assessment.

In this stage, the helpers are urged to conduct a simple but thorough biopsychosocial assessment. This assessment must at least focus on the victim's environmental supports and stressors, medical needs and medication, substance abuse, internal and external methods and resources (Eaton \& Ertl, 2000; Roberts \& Yeager, 2009).

Stage 2: Rapidly establishing rapport. Rapport is facilitated by the helpers. It can be gained through genuineness, respect and acceptance of the client (Roberts, 2005; Roberts \& Yeager, 2009). This stage would install the trust and confidence in victims towards the helpers.

Stage 3: Identify the major problems or crisis precipitants. Assess and determine the dimensions of the crisis. The helpers have to identify any issues and challenges that victims may encounter especially to those who are facing the crisis by providing useful insights into the crisis they are currently facing. Prioritizing in terms of what to work on first, a concept referred to as "looking for leverage", must be put in place (Egan, 2002 ; Roberts \& Yeager, 2009).
Stage 4: Deal with feelings and emotions. This stage is divided by two main aspects. In the first aspect, the helpers should allow victims to express their feelings and to explain their perspectives about the current crisis situation. At this stage, it is important for the helpers to be active listeners and to conduct paraphrasing, the feelings reflection and probing (Egan 2002). The second aspect is introducing challenging responses into the dialogue. If it is appropriately applied, the victims would loosen their maladaptive beliefs and consider other behavioral options.

Stage 5: Generate and explore alternatives. The helpers and victims should explore strategies and options to tackle tensions in positive ways. At this stage, helpers will observe the individuals as being capable and resilient with various potential and alternative sources that can help.

Stage 6: Implement and action plan. The victims should be able to implement some strategies to deal with the pressure. The helpers would identify individuals who support and become a source of reference for the victims.

Stage 7: Follow up. A post-crisis evaluation of the client includes the physical condition, cognitive mastery of the precipitating event, assessment of overall functioning, satisfaction and progress with ongoing treatment and also for any current stressors. The helpers could also determine any need for possible referrals.

According to Roberts (2002), a research on crisis intervention has been conducted to an adolescent potential suicidal attempter. The adolescent barricaded himself in his bedroom and the crisis workers from crisis units helps to calm the depressed adolescent using Roberts's seven stages models. The research concludes that crisis interveners or helpers should be effective by active, directive, focused and helpful. It is critically important for the crisis interveners to work on the seven stages and completely using the approach that mentioned. It's approved that the Roberts seven stages model had been utilized on crisis intervention for helping person in acute psychological crisis, situational crisis or acute stress disorder.

\subsection{The ABC Crisis Intervention Model}

According to Kanel (2012), ABC crisis intervention model is one of the methods to conduct short sessions with clients with functionality that decreases, as an effect from psychosocial stress. The $\mathrm{ABC}$ model is stated below:

The A. Developing and Maintaining Rapport: The helpers have to show attentive behavior that is necessary for building and maintaining good relationship towards victims. The friendly relationship development is one of the key to provide help. When a victim feels comfortable with and trust the helpers, there will be an openness that makes the session's progress continues.

Rapport could be earned by applying some correct attending skills such as listening, responding, clarifying, paraphrasing, reflecting and summarizing.

The B. Identifying the problem: The helpers have to identify the precipitating events, perceptions, subjective distress and functioning that are involved. The most crucial step is to explore the victim's perceptions. Through some exploration work about crucial attending skills, the helpers can gain knowledge and help the victims to identify the source of their pain.

On the other hand, it is suggested that the helpers make some ethical checks on suicidal thoughts, abuse issues, mental health, and substance abuse issues. Using some therapeutic interaction by providing supportive statements, educational information, empowering statement, and reframing statements, the victims can be 
helped to think differently about the situation. These techniques can assist them to cope with the crisis situation.

The C. Coping: The final step is to focus on the coping behavior. Kanel (2012), stated that past coping success could be built on to help the person weather the present and future difficulties. The helpers can help the victims by identifying their own attempts at coping. After further discussion, helpers can encourage the development of new coping behavior. But as for the victims, they are also allowed to propose their own methods of coping as alternative coping behaviors. Having support systems is also one of the coping mechanisms that could help. Rather than having to seek help from mental health professionals, the victims could ask for natural support systems such as from coworkers, relatives, friends and anyone who they are comfortable with.

It is good to recommend crisis victim to seek therapy with trained professionals such as individual therapy or family therapy. Sometimes, a crisis can be seen as an opportunity to resolve any long-term issues that have been hidden before. The helpers also need to be knowledgeable about community agencies and resources. If medical or legal referrals are necessary, then ask their permission to make arrangement for referrals. Lastly, both of the parties have to agree on the commitment and follow up so that they can continue with the helping relationship.

In sum, the $\mathrm{ABC}$ model of crisis intervention is structured short terms approach that guides the helpers through various situations of crisis

\section{Purpose of studies}

The purposes of this study are to identify:

The meaning of crisis among the helpers.

The processes used by the helpers to handle

crisis intervention.

Crisis intervention model used by the helpers.

\section{Methodology}

This research is using a qualitative approach to help in obtaining in-depth and detailed information through semi-structured interviews. Researcher prepared the questions guided by identifies themes in a consistent and systematic manner to elicit more elaborate responses from the interviewer. Semi structured is a flexible accessible and intelligible and capable of disclosing important human or organizational behavior (Qu \& Dumey, 2011). Semi structured interview is the most effective and convenient method of gathering information (Kvale \& Brinkman 2009; Qu \& Dumey, 2011).

The information is gathered from counselors in schools, higher learning institutions and public services. A total of 25 counselors have been selected, and they participated in the semi-structured interviews that employed open-ended questions. The aim was to obtain information on helpers comprehending of crisis, process or strategy that are currently being used by helpers to handle crisis intervention cases and model used by the helpers.

Data from the semi-structured interview method in this research would be analyzed with content analysis. To facilitate the analysis, the information from the interview transferred to the written form obtained through the interview transcripts or notes (Clandinin \& Connelly, 1998). According to Miles and Huberman (1984), qualitative data analysis (interviews) involves namely three processes which are data reduction, data display and formulation and validation of data. This process is to create themes and sub-themes. The number of samples selected in this study is appropriate based on the focus of the study and samples representing the diversity of experience and expertise in the issues studied (Othman, 2007).

\section{Results and discussion}

A total of 25 respondents were involved in this study. They were all registered counselors, 10 males and 15 females. Five of them were employed in schools, 12 were employed at higher education institutions, and eight were from the public services.

\subsection{Meaning of crisis among helpers}

The results of the semi-structured interviewed found that all 25 helpers had been involved in handling of crisis interventions that required them to intervene in the crisis cases faced by individuals.

Overall, the respondents' comprehending of crisis is that "crisis is an event that occurs suddenly, crucial and affects the emotional, spiritual and physical aspects of the individual. Crisis will leads to unstable, confusion and may turn to disaster if individual not resilience enough. It also dangerous and may affect individual and community well-being. The study found that the respondents' comprehending of the crisis were quite accurate and varied depending on the situations experienced.

Among the types of crisis that respondents had ever dealt with were cases involving the death of family members or significant individuals, rape, divorce, extramarital pregnancy, stress, bullying, accidents, suicide attempts, illness (HIV positive), plane crash, fire, natural disasters (floods) and environmental crisis such as career changes.

There are many definitions of crisis. According to James (2008), people can be in a state of crisis when they face an obstacle to important life goals, crisis is a state of disorganization to people who faced frustration of important life goals or in their life circle. The term crisis usually refers to a person's feeling of fear, shock or distress about the situation that they faced (Brammer, 1985; James 2008). Crisis need to be understood clearly by the helpers before dealing with it.

\subsection{Process used by helpers to handle crisis intervention cases}

The results showed that no regular and systematic processes were being used by the helpers while they handled the crisis intervention cases. The helpers depended only on their knowledge and skills of dealing with crisis situations. Most of the helpers stated that the main steps or processes used were a) to identify the problem of the issues and crises faced, b) to stabilize the victims' emotions with the application of relaxation techniques as well as giving space to the clients to share whatever they want to say. c) Another procedure is to establish a good relationship with the clients and d) to ensure that the clients are in a safe and comfortable environment.

Findings from the semi-structured interviews conducted with the helpers indicated that the role of counselors in dealing with crisis cases is as individuals who help to ease emotional distress. In crisis, counselors need to calm the victims, preparing basic needs, provide information and used listening skills to help victims. Psychological First Aid (PFA) is the best technique to use to reduce stress symptoms and assist healthy recovery following a crisis or traumatic event.

As discussed earlier in Roberts' seven stage crisis intervention model and $\mathrm{ABC}$ crisis intervention model, another model on crisis intervention is a six step model by James (2008). James (2008) introduces the six step model that incorporates fundamental counseling skills into a systematic helping process. The model basically focused on facilitative listening and organized skills to the 
emerging feelings, concerns, and situations that clients having most types of trauma may present.

The six step models are as follows. Step 1: explores and define the problems from victims or client's point of view. Step 2: ensures the victims or client's physical and psychological safety. Step 3: provides supports for the person in crisis. Step 4: examines alternative available for victims or clients. Step 5: assists the client in developing a plan of action. Lastly step 6: helps the client to make a commitment and carry out a positive change.

All of the strategies mentioned were important steps to help the helpers handle the crisis interventions. It is serve to organize and simplify the work of the helpers. The results shows that helpers in Malaysia have enough experience in handling crisis cases however still have a lack of knowledge and information that is important for crisis intervention. Therefore there is a need for researchers to come up with a process and strategies that are suitable with Malaysia environment situation in order crisis intervention can be implemented effectively.

\subsection{Crisis intervention model used among helpers}

Only nine out of 25 helpers employed models such as Roberts Seven Stages, Gilliland's Six Steps Model, Kubler Ross Model, Critical Incident Stress Debriefing to guide them to handle crisis intervention. The rest (19 helpers) stated that no model was specifically used in crisis management and they only applied some of the counseling theories such as Cognitive Behavioral Therapy (CBT), Reality Therapy (RT), Psychological First Aid (PFA) and basic attending counseling skills that they perceived to be appropriate to deal with the victim's situation.

This shows that a complete model should be issued to the helpers and training should be implementing in order to intervene in crisis intervention situation. It's supported Lloyd-Evans et.al (2016), that development of fidelity measures for complex intervention in mental health services has been advocated to support service improvement, to define an intervention and measures services' adherence to the model specified.

Therefore, it can be deduced that the crisis handling environment in Malaysia is still at an early stage and has not yet been introduced to the helpers. Most of the responses received stated that the helpers were not ready or have no complete preparation to deal with a crisis. According to the helpers, in Malaysia, there is no uniformity and cooperation between different agencies in dealing with a crisis.

\section{Conclusion}

The comprehending of crisis interventions among helpers in Malaysia is still in its infancy needed to be improved. The helpers need one standardized model and module to attend to crisis cases and conduct the intervention appropriately. According to Nurizien et.al (2010), crisis intervention counseling differs from regular counseling. This is because it requires a counselor to be always ready and open to handle the situation anytime and anywhere. In addition, crisis intervention counseling also lasts within a short period of time and usually does not exceed seven weeks. It helps clients to resolve the crisis they face and put themselves in balance.

A structured model could help the helpers to manage the crisis situations professionally and confidently. Therefore, it is suggested that a model of crisis intervention based on a Malaysian culture needs to be developed as a guideline for the helpers in dealing with event of crisis.

\section{Acknowledgment}

This research received funding from the Fundamental Research Grant Scheme (FRGS), Ministry of Higher Education Malaysia (203.PGURU.6711548).

\section{References}

[1] Brammer,L.M.(1985).The helping relationship: process and skills (3rd ed). Upper sadle River, Nj:Prentice Hall. In James, R. K. 2008. Crisis intervention strategies (6th ed.). Belmont, CA: Thomson Brooks/Cole.

[2] Clandinin, J.D., \& Connelly, F. M. (1998). Personal experience methods In . Denzin, Norman, K. \& Lincoln, Yvonna, S (pnyt.). Collecting and interpreting qualitative materials, 413-427. California: Sage Publications Inc.

[3] Egan, G. (2002). The skilled helpers (7thed.). Belmont, CA: Wadsworth

[4] Eaton, Y., \& Ertl, B. (2000). The comprehensive crisis intervention model of community integration, Inc. crisis services. In Roberts, A.R.,\& Yeager, K.R. Pocket guide of crisis intervention.(pp. 40-41) Oxford University Press.

[5] Ehsan Khodarami.(2009). Crisis management, disaster prevention and management : An international Journal. Vol.18.Issue 5.Pp.523-528. Doi.10.1108/ 09653560911003714

[6] Greenstone, J.L.,\& Leviton, S.C. (2002). Elements of crisis intervention: crises and how to respond to them (2nd Ed). Belmont, CA: Thomson Brooks/Cole.

[7] Greenstone, J.L.,\& Leviton, S.C. (2011). Elements of crisis intervention: crises and how to respond to them (3rd Ed). Belmont, CA: Thomson Brooks/Cole.

[8] James, R. K.,. \& Gilliland, B. E. (2005). Crisis intervention Strategies (5th Ed.). Belmont, CA: Thomson Brooks/Cole.

[9] James, R. K. (2008). Crisis intervention strategies (6th Ed.). Belmont, CA: Thomson Brooks/Cole.

[10] Kanel,.K. (2012). A Guide to Crisis Intervention. CengageLearning.

[11] Kvale.,S.,\& Brinkman.,S.(2009). Interviews: Learning the craft of qualitative reseach interviewing. SAGE.Los Angeles.C.A in Qu.,S.Q.,\& Dumay.,J. The qualitative research interview, qualitative research in accounting and management. Vol.8 Issues:3. Pp. 238 - 264. Doi.0rg/ 10 101108 / 111766091111162070.

[12] Lloyd-Evans.B., Bond.R.G., Ruud.t., Ivanecka.A.,Gary.R., Osborn.D., Nolan.F., Henderson.C., Mason.O., Goater.N., Kelly.K., Ambler.G., Morant.N., Onyett.S., Lamb.D., Fahmy.S., Brown.E., Paterson.B., Sweeney.A., Hindle.D.,Fullarton.K., Frerichs.J., \& Jonhson.S. (2016) Development of a measure of model fidelity for mental health crisis resolution teams. BMC psychiatry. 16:427. Doi 10.1186/s12888-016-11394.

[13] Miles, M.B., \& Huberman, A.M. (1984). Qualitative data analysis: a sourcebook of new methods. Newbury Park: Sage Publications.

[14] Myer, R.A., \& Conte, C. (2006). Assessment for crisis intervention. Journal of Clinical Psychologist. Vol 62 (8), 959-970. Doi $10.1002 /$ jclp.20282.

[15] Nor Shafrin Ahmad. (2009). Pengenalan kepada Konflik, Amrah, Stress dan Krisis dalam Organisasi (in Adaptasi Pementoran dalam Organisasi). Pulau Pinang: Penerbit Universiti Sains Malaysia.

[16] .Nurizien Binti Shaharudin, Norbaisatul Omar, Faiz Fazana Hamid, Nurhany Binti Abd Rahman,Nurhafizah Binti Lois, Noor Aini Binti Abd Radin \& Siti Hamizah Binti Robian. (2010). Intervensi Krisis. Tajung Malim: Universiti Perguruan Sultan Idris

[17] Othman Lebar. (2007). Penyelidikan Kualitatif: Pengenalan kepada teori dan Metod. Tanjong Malim: Universiti Perguruan Sultan Idris.

[18] Qu.,S.Q.,\& Dumay.,J.(2011). The qualitative research interview, qualitative research in accounting and management. Vol.8 Issues:3. Pp.238264.Doi.Org/ 10.101108/111766091111162070.

[19] Roberts, A.R.,\& Yeager, K.R. (2009). Pocket guide of crisis intervention. Oxford University Press.

[20] .Roberts, A.R. (2005). An overview of crisis theory and crisis intervention. In Roberts, A.R.,\& Yeager, K.R.. Pocket guide of crisis intervention.(pp.42) Oxford University Press.

[21] Roberts, A.R.,(2002). Assessment, crisis intervention, and trauma treatment: the integrative ACT intervention model. Oxford University Press.

[22] Shamsiah Mohd Jais. (2016). Assessment and intervention in crisis :an application of the six step model and triage assessment system. Pakistan Journal of Social Sciences. 13 (1): 1-7. ISSN:1683-8831.Medwell Journals. 